\title{
An Expert System to Detect Polycystic Ovary Syndrome under Uncertainty
}

\author{
Md. Khaliluzzaman ${ }^{1}$, Md. Shahedul Islam², Rezaul Karim ${ }^{3}$ \\ ${ }^{I}$ Dept. of Computer Science \& Engineering, International Islamic University Chittagong (IIUC), Bangladesh \\ ${ }^{2}$ Dept. of Computer Science \& Engineering, Stamford University Bangladesh (SUB), Bangladesh \\ ${ }^{3}$ Dept. of Computer Science \& Engineering, University of Chittagong (CU), Bangladesh
}

\begin{abstract}
This paper describes a prototype of clinical expert system for risk stratification of patients with polycystic ovary syndrome (PCOS). Polycystic ovary syndrome (PCOS) is the most common hormonal disorder among women of reproductive age. It is a heterogeneous disorder of uncertain causes. Since the symptoms of PCOS are seemingly unrelated to one another the condition is often overlooked and undiagnosed. The determination of accurate degree or intensity of PCOS signs is difficult for the physician. Hence, the accuracy of diagnostic process is difficult to achieve. The signs and symptoms of PCOS are usually expressed in qualitative and quantitative ways. Since the qualitative factors can not measured in a quantitative way, various types of uncertainties may occurs such as incompleteness, vagueness, and imprecision. For that, it is necessary to address the issue of uncertainty by using appropriate methodology. However, no existing system is able to address this issue of uncertainty. Therefore, this paper demonstrates the application of a novel method, named belief rule-based inference methodology-RIMER; this prototype can deal with uncertainties in both clinical domain knowledge and clinical data. This paper reports the development of a Belief Rule Based Expert System (BRBES) using RIMER approach, which is capable of detect the PCOS by taking account of signs and symptoms.
\end{abstract}

Keywords: Belief Rule Base Expert System (BRBES), Uncertainty, RIMER, Evidential Reasoning, Polycystic ovary syndrome (PCOS), Signs and Symptoms

\section{Introduction}

PCOS is a common complex condition in women associated with reproductive and metabolic features. It is a chronic disease with manifestations across the lifespan and represents a major health and economic burden. A common ovulation problem that affects about 5\% to $10 \%$ of women in their reproductive years is polycystic ovary syndrome (PCOS). PCOS is a hormonal imbalance that can make the ovaries stop working normally. In most cases, the ovaries become enlarged and appear covered with tiny, fluid-filled cysts. Infertility is one of the most common PCOS symptoms. Because the symptoms of PCOS are seemingly unrelated to one another, the condition is often overlooked and undiagnosed. The determination of accurate degree or intensity of PCOS signs is difficult for the physician. Hence, there is a risk of having incomplete information to reach a conclusion through PCOS diagnosis. Therefore, the accuracy of diagnostic process is difficult to achieve. Since the PCOS symptoms are subjective in nature, it inherits uncertainty. Therefore, it can be seen that the determination of signs and symptoms in a quantitative way is difficult to achieve. Traditional system is used in PCOS diagnosis to detect the disease. But this system is not worked in uncertain. So, ultimately the accuracy in the disease detection process is hampered. As human life is directly involve with medical diagnosis process, disease diagnosis process accuracy is very important factor for saving human life.

There is no specific test to definitively diagnose polycystic ovary syndrome. The diagnosis is one of exclusion, which means doctor considers all signs and symptoms and then rules out other possible disorders. Uncertainty exists in almost every stage of a diagnosis process. Sources of uncertainties may include that patients can't describe exactly what has happened to them or how they feel, doctors and nurses cannot tell exactly what they observe, and laboratories report results may be with some degrees of error. physiologists do not precisely understand how the human body works, medical researchers can't precisely characterize how diseases alter the normal functioning of the body, pharmacologists do not fully understand the mechanisms accounting for the effectiveness of drugs, and no one can precisely determine one's prognosis.

Researchers and scientists have built and applied varies methods in this growing research fields. For uncertainty selection RIMER is treated as appropriate method to solve the certain problems [13][14]. In [7], ER deals with problems under various uncertainties such as incomplete information, vagueness, and ambiguity consisting of both quantitative and qualitative criteria. In particular utility theory the ER approach is developed based on decision theory [1][11], artificial intelligence in particular the theory of evidence [9][10]. A belief 
structure is used to model a judgment with uncertainty. Some linguistic referential value such as excellent, average, good, and bad are used to evaluate qualitative attribute such as location or safety [20][21]. In this way, the issue of uncertainty can be addressed more accurately and robustly during decision made. The belief rule based inference methodology-RIMER [15] has addressed such issue by proposing a belief structure which assigns degree of belief in the various referential values of the attributes.

Consequently, traditional diagnosis, carried out by a physician, is unable to deliver desired accuracy. Moreover, traditional PCOS diagnosis is time consuming and costly. Hence, this paper presents the design, development and application of an expert system that will diagnosis PCOS precisely in a short time with low cost.

In Section II briefly described the belief rule base inference methodology-RIMER. In Section III demonstrated the application of BRB to diagnosis PCOS. In the next Section results and achievements are represented. Finally, the paper is concluded in Section IV.

\section{RIMER To Develop BRBES}

In RIMER, Belief Rule Base (BRB) can capture complicated nonlinear causal relationships between antecedent attributes and consequents, which are not possible in traditional IF-THEN rules. BRB is used to model domain specific knowledge under uncertainty, and the ER approach is employed to facilitate inference. This section introduces $\mathrm{BRB}$ as a knowledge representation schema under uncertainty as well as inference procedures of RIMER.

\section{A. Modeling domain knowledge using BRB}

Belief Rules are the key constituents of a BRB, which include belief degree. This is the extended form of traditional IF-THEN rules. In a belief rule, each antecedent attribute takes referential values and each possible consequent is associated with belief degrees [15]. The knowledge representation parameters are rule weights, attribute weights and belief degrees in consequent attribute, which are not available in traditional IFTHEN rules. A belief rule can be defined in the following way.

$$
R_{k}:\left\{\begin{array}{l}
I F\left(p_{1} \text { is } A_{1}^{k}\right) \cap\left(p_{2} \text { is } A_{2}^{k}\right) \cap \ldots \ldots \ldots\left(p_{T_{k}} \text { is } A_{T_{k}}^{k}\right) \\
\operatorname{THEN~}\left\{\left(C_{1}, \beta_{1 k}\right),\left(C_{2}, \beta_{2 k}\right), \ldots \ldots \ldots,\left(C_{N}, \beta_{N k}\right)\right\} \\
R_{k}:\left(\beta_{j k} \geq 0, \sum_{j=1}^{N} \beta_{j k} \leq 1\right) \text { with a rule weight } \theta_{k} \text { attribute. }
\end{array}\right.
$$

Weights $\delta_{k 1}, \delta_{k 2}, \delta_{k 3}, \ldots \ldots, \delta_{k T_{k}}, k \in\{1, \ldots \ldots, L\}$

Where $p_{1}, p_{2}, p_{3} \ldots p_{T_{k}}$ represent the antecedent attributes in the $k^{\text {th }}$ rule. $A_{i}^{k}\left(i=1, \ldots \ldots, T_{k}, k=\right.$ $1, \ldots \ldots, L$ represents one of the referential values of the $i^{\text {th }}$ antecedent attribute $P_{i}$ in the $k^{\text {th }}$ rule. $C j$ is one of the consequent reference values of the belief rule.

$\beta_{j k}(j=1, \ldots \ldots, N k=1, \ldots \ldots, L)$ is one of the the belief degrees to which the consequent reference value $C_{j}$ is believed to be true. If

$\sum_{j=1}^{N} \beta_{j k}=1$

is the $k^{\text {th }}$ rule is said to be complete. Otherwise, it is incomplete. $T_{k}$ is the total number of antecedent attributes used in $k^{\text {th }}$ rule $L$ is the number of all belief rules in the rule base. $N$ is the number of all possible consequent in the rule base. For example a belief rule to assess Metabolic syndrome (A9) for PCOS can be written in the following way.

$\mathrm{R}_{\mathrm{k}}$ : A2 is $\mathrm{H}^{\wedge} \mathrm{A} 3$ is $\mathrm{H}^{\wedge} \mathrm{A} 4$ is $\mathrm{H}^{\wedge} \mathrm{A} 5$ is $\mathrm{H}$ THEN A9 is $\{((\mathrm{H}, 1.0),(\mathrm{M}, 0.0),(\mathrm{L}, 0.0)\}$

Where $\{(\mathrm{H}$ (High), 1.0), (M (Medium), 0.0), (L (Low), 0.0)\} is a belief distribution for A9 (masculinizing hormons) consequent, stating that the degree of belief associated with High is $100 \%, 0 \%$ with medium and $0 \%$ with low. In this belief rule, the total degree of belief is $(1.0+0.0+0.0)=1$, hence that the assessment is complete.

\section{B. BRB Inference using ER}

The ER approach [7] [18] developed to handle multiple attribute decision analysis (MADA) problem having both qualitative and quantitative attributes. Different from traditional MADA approaches, ER presents MADA problem by using a decision matrix, or a belief expression matrix, in which each attribute of an alternative described by a distribution assessment using a belief structure. The inference procedures in BRB inference system consists of various components such as input transformation, rule activation weight calculation, rule update mechanism, followed by the aggregation of the rules of a BRB by using ER [15][16][18]. The input transformation of a value of an antecedent attribute $P_{i}$ consists of distributing the value into belief degrees of different referential values of that antecedent. This is equivalent to transforming an input into a distribution on referential values of an antecedent attribute by using their corresponding belief degrees [14]. The $i^{\text {th }}$ value of an antecedent attribute at instant point in time can equivalently be transformed into a 
distribution over the referential values, defined for the attribute by using their belief degrees . The input value of $P_{j}$, which is the $\mathrm{i}^{\text {th }}$ antecedent attribute of a rule, along with its belief degree $\varepsilon_{i}$ is shown below by Eq. (3). The belief degree $\varepsilon_{i}$ to the input value is assigned by the expert in this research.

$H\left(P_{i}, \varepsilon_{i}\right)=\left\{\left(A_{i j}, \alpha_{i j}\right), j=1, \ldots, j_{i}\right\}, i=1, \ldots \ldots, T_{k}$

Here, $\mathrm{H}$ is used to show the assessment of the belief degree assigned to the input value of the antecedent attribute. In the above equation $A_{i j}$ ( $i^{\text {th }}$ value) is the $j^{\text {th }}$ referential value of the input $P_{i} \cdot \alpha_{i j}$ is the belief degree to the referential value $A_{i j}$ with $\alpha_{i j} \geq 0 . \sum_{j=1}^{j_{1}} \alpha_{i j} \leq 1\left(i=1, \ldots, T_{k}\right)$, and $j_{i}$ is the number of the referential values.

For example, the input 0.92 for masculinizing hormones is equivalently transformed to $\{(\mathrm{High}, 0.87)$, (Medium,0.11), (Low, 0.02)\}.The input value of an antecedent attribute is collected from the expert in terms of linguistic values such as 'High', 'Medium', and 'Low'. This linguistic value is then assigned degree of belief $\varepsilon_{i}$ by taking account of expert judgment. This assigned degree of belief is then distributed in terms of belief degree $\alpha_{i j}$ of the different referential values $A_{i j}$ [High, Medium, Low] of the antecedent attribute. The above procedure of input transformation is elaborated by equations (4 and 5) given below. However, it is important for us to know, with what degree of belief it is High and with what degree of belief it is Medium. This phenomenon can be calculated with the following Eq. (4) and Eq. (5).

$\beta_{n, i}=\frac{h_{n+1}-h}{h_{n+1, i}-h_{n, i}}, \beta_{n+1, i}=1-\beta_{n, i}$

If $h_{n, i} \leq h \leq h_{n+1, i}$

Here, the degree of belief $\beta_{n, i}$ is associated with the evaluation grade Low while $\beta_{n+1, i}$ is associated with the upper level evaluation grade i.e. High.

When the $k^{\text {th }}$ rule is activated, the weight of activation of the $k^{\text {th }}$ rule, $w_{k}$ is calculated by using the flowing formula define in Eq. (6) [17] [18].

$w_{k}=\frac{\theta_{k} \alpha_{k}}{\sum_{j=1}^{L} \theta_{j} \alpha_{j}}=\frac{\theta \prod_{i=1}^{T_{k}}\left(\alpha_{i}^{k}\right)^{k} \bar{\delta}_{k i}}{\sum_{j=1}^{L} \theta_{j}\left[\prod_{l=1}^{T}\left(\alpha_{l}^{k}\right)^{\delta_{k l}}\right]}$

Here,

$\bar{\delta}_{k l}=\frac{\delta_{k i}}{\max _{i=1, \ldots ., T_{k}}\left(\delta_{k i}\right)}$

Where $\bar{\delta}_{k l}$ is the relative weight of $P_{i}$ used in the $k^{\text {th }}$ rule, which is calculated by dividing weight of $P_{i}$ with maximum weight of all the antecedent attributes of the $\mathrm{k}^{\text {th }}$ rule. By doing so, the value of $\bar{\delta}_{k l}$ becomes normalize, meaning that the range of its value should be between 0 and $1 . \alpha_{k}=\prod_{i=1}^{T_{k}}\left(\alpha_{i}^{k}\right)^{\overline{\delta_{k i}}}$ is the combined matching degree, which is calculated by using multiplicative aggregation function.

When the $k^{\text {th }}$ rule as given in Eq. (1) is activated, the incompleteness of the consequent of a rule can also result from its antecedents due to lack of data. An incomplete input for an attribute will lead to an incomplete output in each of the rules in which the attribute is used. The original belief degree $\bar{\beta}_{i k}$ in the $i^{\text {th }}$ consequent $C_{i}$ of the $k^{\text {th }}$ rule is updated based on the actual input information as define in Eq. (7) [15][17][18].

$\beta_{i k}=\bar{\beta}_{i k} \frac{\sum_{t=1}^{T_{k}}\left(\tau(t, k) \sum_{j=1}^{j} \alpha_{t j}\right)}{\sum_{t=1}^{T_{k}}(\tau(t, k)}$

Where

$\begin{aligned}(t, k)= & \left\{\begin{array}{l}1, \text { if } P_{i} \text { is used in defining } R_{k}\left(t=1, \ldots \ldots, T_{k}\right) \\ 0, \text { otherwise }\end{array}\right. \\ & \text { Here } \bar{\beta}_{i k} \text { is the original belief degree and } \beta_{i k} \text { is the updated belief degree. }\end{aligned}$

Due to the incomplete input for "masculinizing hormons", the belief degree of the connected rules needs to be modified to show the incompleteness by using Eq. (7).

$\beta_{i k} \equiv \bar{\beta}_{i k} \frac{1.6}{2}=\bar{\beta}_{i k} * 0.8, \quad i=1,2,3 ; k=1, \ldots \ldots, 9$

Therefore $0<\sum_{i=1}^{3} \beta_{i k}<1$ for all rules that are associated with "masculinizing hormons". Using the sub rule base, the assessment result for "masculinizing hormons" is obtained using BRBES system : $\{$ (High, 0.66), (Medium ,0.23), (Low, 0.02), (Unknown, 0.09) $\}$ where Unknown in the above result means that the output is also incomplete input. ER approach is used to aggregate all the packet antecedents of the $L$ rules to obtain the degree of belief of each referential values of the consequent attribute by taking account of given input values $P_{i}$ of antecedent attributes. This aggregation can be carried out either using recursive or analytical approach. In this research analytical approach [19] has been considered since it is computationally efficient than recursive approach [14] [20] [21], because analytical approach deal with all parameter such as rule weight, attribute weight, belief degree, utility etc. For this why there is no chance of absence of any 
parameter. The conclusion $O(Y)$, consisting of referential values of the consequent attribute, is generated. Eq. (8) as given below illustrates the above phenomenon.

$$
O(Y)=S\left(P_{i}\right)=\left\{\left(C_{j}, \beta_{j}\right), j=1,2, \ldots \ldots, N\right\}
$$

Where, $\beta_{j}$ denotes the belief degree associated with one of the consequent reference values such as $C_{j}$ and $\beta_{j}$ is calculating by analytical format of the ER algorithm [3] as illustrated in equation (9).

$\beta_{j}=\frac{\mu\left[\prod_{k=1}^{L}\left(\left(w_{k} \beta_{j k}+1-w_{k} \sum_{j=1}^{N} \beta_{j k}\right)\right)-\prod_{k=1}^{L}\left(1-w_{k} \sum_{j=1}^{N} \beta_{j k}\right)\right]}{1-\mu\left[\prod_{k=1}^{L} 1-w_{k}\right]}$

With

$$
\mu=\left\lceil\sum_{j=1}^{N} \prod_{k=1}^{L}\left(w_{k} \beta_{j k}+1-w_{k} \sum_{j=1}^{N} \beta_{j k}\right)-(N-1) \prod_{k=1}^{n}\left(1-w_{k} \sum_{j=1}^{N} \beta_{j k}\right)\right]^{-1}
$$

The final combined result or output generated by $\mathrm{ER}$ is represented by $\left\{\left(C_{1}, \beta_{1}\right),\left(C_{2}, \beta_{2}\right), \ldots \ldots,\left(C_{N}, \beta_{N}\right)\right\}$. Here $\beta_{j}$ is the final belief degree attached to the $j^{\text {th }}$ referential value $C_{j}$ of the consequent attribute, obtained after combining all activated rules in the BRB by using ER.

\section{Output of the BRB System}

The output of the BRB system is not crisp/numerical value. Hence, this output can be converted into crisp/numerical value by assigning utility score to each referential value of the consequent attribute [17].

$$
H\left(A^{*}\right)=\sum_{j=1}^{N} u\left(C_{j}\right) B_{j}
$$

Where, $H\left(A^{*}\right)$ is the expected score expressed as numerical value and $u\left(C_{j}\right)$ is the utility score of each referential value. For example, in this paper the overall assessment result is $\{(\mathrm{H}, 0.55),(\mathrm{M}, 0.25),(\mathrm{L}, 0.20)\}$ for PCOS disease, then the expected utility score is 0.675 or $68 \%$ which represents Medium risk disease. In this paper the RIMER methodology to address various type of uncertainty such as incompleteness, ignorance and impreciseness by using Eq. (7) and Eq. (11).The incompleteness as mentioned occurs due to ignorance, meaning that belief degree has not been assigned to any specific evaluation grade and this can be represented using the equation as given below.

$$
\beta_{H}=1-\sum_{n=1}^{N} \beta_{n}
$$

Where, $\beta_{H}$ is the belief degree unassigned to any specific grade. If the value of $\beta_{H}$ is zero then it can argued that there is an absence of ignorance or incompleteness. If the value of $\beta_{H}$ is greater than zero then it can be inferred that there exists ignorance or incompleteness in the assessment.

$\beta_{i k}=\bar{\beta}_{i k} \frac{\sum_{t=1}^{T_{k}}\left(\tau(t, k) \sum_{j=1}^{j t} \alpha_{t j}\right)}{\sum_{t=1}^{T_{k}}(\tau(t, k)}$

\section{BRBES Architecture}

Architectural design represents the structure of data and program components that are required to build a computer-based system. It is also considers the pattern of the system organization, known as architectural style. BRBES adopts the three-layer architecture, which consisting of presentation, application and data processing layer is as shown in Fig. 1.

\section{A. System Components}

The input clarifications of input antecedent are:

A $1=$ menstrual disorders,

$\mathrm{A} 2=$ acne

A3=hirsutism

A4= hyperorrheamen

A5 $=$ androgenic alopecia

A6 $=$ Central obesity

A7 $=$ Insulin resistance are transformed to referential 


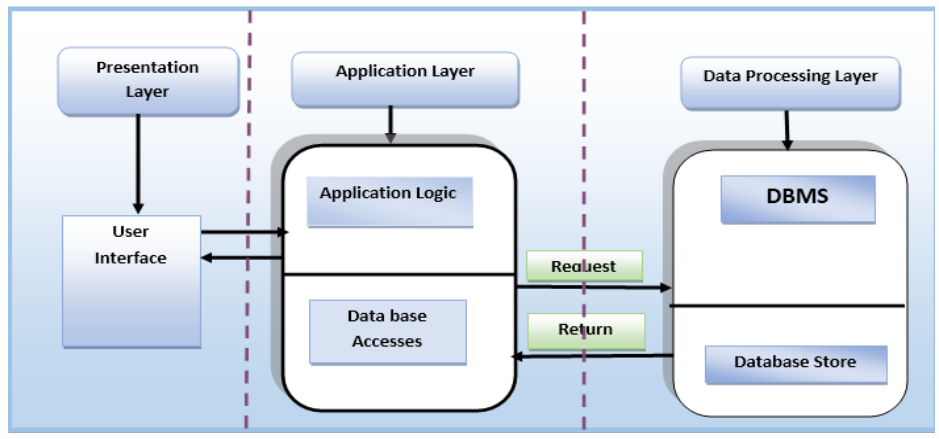

Fig. 1. BRBES Architecture.

Value is evaluated by Eq. (4), (5) on behalf of expert. The input clarifications of this BRB system transformed to referential is shown in Table I.

Table I. The Input Are Transformed In Referential Value

\begin{tabular}{|c|c|c|c|c|c|}
\hline Sl. No. & Input Antecedent & Expert Belief & \multicolumn{3}{|c|}{ Referential Value } \\
\hline & & & High & Medium & Low \\
\hline 0 & A1 & 1.0 & 1 & 0 & 0 \\
\hline 1 & A2 & 0.5 & 0.1 & 0.7 & 0.2 \\
\hline 2 & A3 & 0.8 & 0.5 & 0.5 & 0 \\
\hline 3 & A4 & 0.5 & 0.1 & 0.8 & 0.1 \\
\hline 4 & A5 & 1 & 0.8 & 0.2 & 0 \\
\hline 5 & A6 & 0.5 & 0.1 & 0.4 & 0.5 \\
\hline 6 & A7 & 1 & 0.8 & 0.2 & 0 \\
\hline
\end{tabular}

\section{B. Knowledge Base Constructed using BRB}

In present paper, we worked on polycystic ovary syndrome (PCOS). In order to construct BRB knowledge base of this system we designed a BRB framework to PCOS diagnosis according to clinical domain expert (doctor).The BRB framework of PCOS diagnosis as illustrated in Figure 2, From the framework, it can be observed that input factors that determine this disease level. The BRB knowledge base has different traditional rule to assessment, which need to convert brief rules.

In such situations, belief rules may provide an alternative solution to accommodate different types and degrees of uncertainty in representing domain knowledge. A BRB can be established in the following four ways[15]- (1) Extracting belief rules from expert knowledge (2) Extracting belief rules by examining historical data, (3) Using the previous rule bases if available, and (4) Random rules without any pre-knowledge.

In this paper, we constructed initial BRB by the domain expert knowledge. There will be four sub-rule bases, which can be named as follows:

1) A8 sub-rule-base

2) A9 sub-rule-base

3) A10 sub-rule-base

4) A11 sub-rule-base

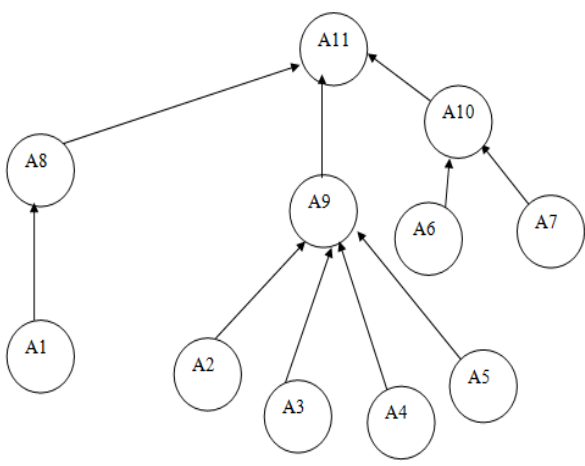

Fig. 2. Hierarchical Relationship among PCOS diagnosis variables.

The Calculation of the initial rule-base for various sub-rule-bases

Initial rule-base for A8 which will be consisting of 3 rules

Initial rule-base for $\mathrm{A} 9$ which will be consisting of 81 rules

Initial rule-base for A10 which will be consisting of 9 rules 
Initial rule-base for A11 which will be consisting of 27 rules

The entire BRB consists of $(3+81+9+27)=120$ belief rules. It is assumed that all belief rules have equal rule weight; all antecedent equal weight, and the initial belief degree assigned to each possible consequent by two expert from accumulated the data. To better handle uncertainties, each belief rule considered the three referential values are High $(\mathrm{H})$, Medium (M) and Low (L)

Table II: Initial Belief Rules of Sub-Rule-Base (Metabolic syndrome)

\begin{tabular}{|c|c|c|c|c|c|c|}
\hline \multirow{2}{*}{$\begin{array}{c}\text { Rule } \\
\text { No. }\end{array}$} & \multirow{2}{*}{$\begin{array}{c}\text { Rule } \\
\text { Weight }\end{array}$} & \multicolumn{2}{|c|}{ IF } & \multicolumn{4}{c|}{ THEN } \\
\cline { 3 - 7 } & A6 & A7 & \multicolumn{2}{c|}{ A10(Metabolic syndrome) } \\
\hline & & & & High & Medium & Low \\
\hline 0 & 1 & H & H & 0.8 & 0.2 & 0 \\
\hline 1 & 1 & H & M & 0.4667 & 0.5333 & 0 \\
\hline 2 & 1 & H & L & 0.0667 & 0.9333 & 0 \\
\hline 3 & 1 & M & H & 0 & 0.9333 & 0.1 \\
\hline 4 & 1 & M & M & 0 & 0.8 & 0.2 \\
\hline 5 & 1 & M & L & 0 & 0.6667 & 0.3 \\
\hline 6 & 1 & L & H & 0.3333 & 0.6667 & 0 \\
\hline 7 & 1 & L & M & 0 & 0.9333 & 0.1 \\
\hline 8 & 1 & L & M & 0 & 0.8 & 0.2 \\
\hline
\end{tabular}

\section{Inference Engine using ER}

This BRBES designed using the ER approach [15] [18] [20] [21] which is described in section II (B). It is similar to traditional forward chaining. The inference with a BRB using the ER approach also involves assigning values to attributes, evaluating conditions and checking to see if all of the conditions in a rule are satisfied. The BRB inference process using the ER approach described by the following steps are input transformation, calculation of the activation weight, calculating combined belief degrees to all consequents, belief degree update and aggregate multiple activated belief rules. The inputs of data are of two types, objective and subjective. Input transformation of this system and input clarification are deduced in previous section and Table I by using Eq. (4) and Eq. (5). After the value assignment for antecedent, calculating the combined matching degrees between the inputs and the rule's antecedents, the next step is to calculate activation weight for each packet antecedent in the rule base using Eq. (6). The belief degrees in the possible consequent of the activated rules in the rule base are updated using Eq. (7). Then aggregating all activated rules using the ER approach to generate a combined belief degree in possible consequents using Eq. (8) and Eq. (9). Then expected result of PCOS diagnosis was calculated from its different consequents of factors. Finally, presenting the system inference results of PCOS diagnosis consequent which is not crisp/numerical value, then it is converted into crisp/numerical value for recommendation using Eq. (10).

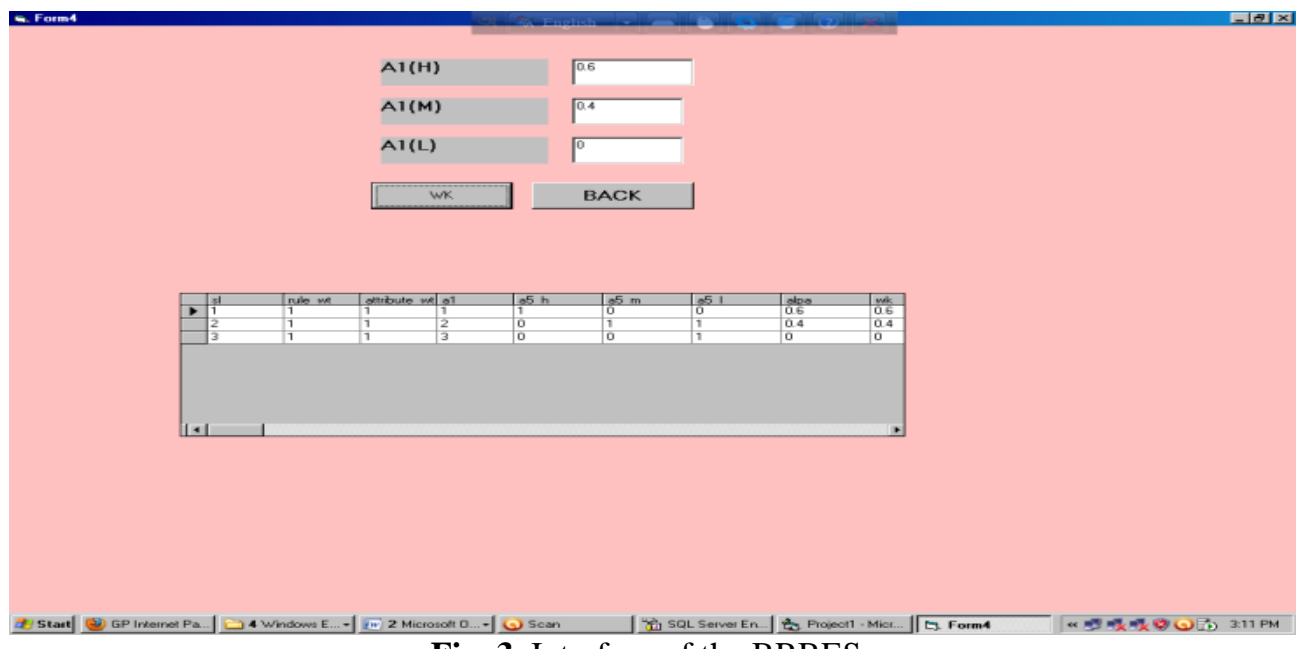

Fig. 3. Interface of the BRBES.

\section{BRB ES Interface}

System interface is an intermediate position that represents the interaction between user and system. Fig. 3 represents the BRB system interface.

\section{2) Results and Discussion}


In this paper, the clinically simulated data set variables are used to determine PCOS the one patient's clinical signs, symptoms, and PCOS include namely menstrual disorders, acne, hirsutism, hyperorrheamen, androgenic alopecia, Central obesity, Insulin resistance, A11(PCOS) A8 (infertality), (A9) masculinizing hormons, (A10) Metabolic syndrome. Where the PCOS is dependent variable, it is used to present outcome as PCOS present or not, if PCOS diagnosis score result is greater than 70\%, it indicates the PCOS is High. If the score result is less than $70 \%$ it indicates the PCOS is Medium otherwise Low. The real data set of the 36 patients were collected and simulated having PCOS with different clinical level. The eight patient's simulated data set with diagnosis outcome is presented as example in Fig. 4. This figure represents overall PCOS diagnosis outcome from patient's information. The result of this system is measured in percentage for recommendation. The output of this system was generated based on output utility Eq. (10). In this paper, the utility score of $100 \%$ assigned to 'High', $50 \%$ assigned to 'Medium', and $0 \%$ assigned to 'Low'. For example, we can estimate overall system output PCOS as 99\%, if the Fuzzy result of the system is ( High, 0.90), (Medium, 0.10), (Low, 0)\}.

In the case study, the PCOS of 36 patients using this system, doctors' manual system and clinical history result is shown in Fig. 4. The clinical historical results were considered as benchmark. From Fig. 4 it can be observed that ES generated result has less deviation than from benchmark result. Hence, it can be argued that ES output is more reliable than manual system. Therefore, it can be concluded that if the assessment of PCOS is carried out by using the ES, eventually this will play an important role in taking decision to avoid unnecessary costly lab investigation.

\begin{tabular}{|l|l|l|l|l|l|l|l|l|l|l|l|l|}
\hline Patient ID & Age & A1 & A2 & A3 & A4 & A5 & A6 & A7 & Manuel Result & Benchmark Result & BRB System Result & Risk Stage \\
\hline 1 & 50 & H & H & H & H & H & M & L & $80.44 \%$ & $92.55 \%$ & $91.55 \%$ & High $>70 \%$ \\
\hline 2 & 67 & M & L & M & H & M & L & M & $67.33 \%$ & $69.55 \%$ & $66.99 \%$ & Medium< $<70 \%$ \\
\hline 3 & 47 & L & H & M & L & M & H & H & $57.55 \%$ & $50 \%$ & $52.66 \%$ & Low<50\% \\
\hline 4 & 52 & M & M & H & L & H & M & L & $85 \%$ & $90 \%$ & $87 \%$ & High $>70 \%$ \\
\hline 5 & 40 & L & L & L & M & L & L & H & $52.11 \%$ & $55.23 \%$ & $54 \%$ & Low<50\% \\
\hline 6 & 53 & H & H & H & H & H & L & M & $85 \%$ & $90 \%$ & $87 \%$ & High $>70 \%$ \\
\hline 7 & 36 & M & L & M & H & M & Y & L & $52.11 \%$ & $43.23 \%$ & $45 \%$ & Low<50\% \\
\hline 8 & 63 & L & H & M & L & M & L & M & $61.66 \%$ & $67.43 \%$ & $69.88 \%$ & Medium< $<70 \%$ \\
\hline
\end{tabular}

Fig. 4. Simulated Data by BRBES (H-High, M-Medium, L-Low).

\section{3) Conclusion}

The development and application of a belief rule based Expert system (BRBES) to detect Polycystic Ovary Syndrome (PCOS) by using signs and symptoms of patients have been presented. The prototype of ES is embedded with a novel methodology known as RIMER. Which allows the handling of various types of uncertainty and hence be considered as a robust tool can be utilized in detecting PCOS. Consequently, the prototype of ES can handle various types of uncertainties found in clinical domain knowledge as well as in signs and symptoms of a patient. Most importantly, the system will play an important role in reducing the cost of lab investigations. The system will facilitate patients in taking precautionary measures well in advance. It can also provide a percentage of risk recommendation, which is more reliable and informative than from the traditional expert's opinion. The prototype of ES can only be used to detect PCOS by using signs and symptoms of a patient.

In further research will consider the PCOS accurate detection system using signs and symptoms. The validation of the system is necessary by using real patient historical data and the knowledge representation parameters should be trained by real clinical data using the BRB optimal learning training model. At last, the employing of RIMER to develop BRBES for PCOS suspicion is a valid new approach and real data can be used to training in future research.

\section{Acknowledgment}

The authors are grateful to Dr. Mumtahena Mahmuda MBBS (DMC), FCPS, BCS (Health) working as the medical officer of government Hospital for providing domain knowledge and support in this research.

\section{References}

[1] M Sonmez, G. Graham and J. B. Yang and G D Holt, "Applying evidential reasoning to pre-qualifying construction contractors", Journal of Management in Engineering, Vol.18, No.3, pp.111-119, 2002.

[2] J. B. Yang, "Rule and utility based evidential reasoning approach for multiple attribute decision analysis under uncertainty", European Journal of Operational Research, Vol. 131, No.1, pp.31-61, 2001.

[3] Y. M. Wang, J. B. Yang and D. L. Xu, "Environmental Impact Assessment Using the Evidential Reasoning Approach", European Journal of Operational Research, Vol.174, No.3, pp.1885-1913, 2006.

[4] Lisa M. (2008). The Sage encyclopedia of qualitative research methods. Los Angeles, Calif.: Sage Publications. ISBN 1-41294163-6.

[5] http://www.pearson.ch/1449/9780273722595/An-Introduction-to-Geographical.aspx.

\begin{tabular}{|c|c|}
\hline $3008-1104013643$ & www.iosriournals.org \\
\hline
\end{tabular}


[6] Dodge Y. (2003) The Oxford Dictionary of Statistical Terms, OUP. ISBN 0-19-920613-9.

[7] D. L. Xu and J. B. Yang, "Introduction to multi-criteria decision making and the evidential reasoning approach", Working Paper Series,Paper No.: 0106 , ISBN: 186115111 X (http://www.umist.ac.uk/management), Manchester School of Management, UMIST, pp. 1-21, 2001.

[8] A. Taroun and J. B. Yang. "Dempster-Shafer theory of evidence: potential usage for decision making and risk analysis in construction project management." Journal of the Built and Human Environment Review 4, no. 1(2011) : 155-166

[9] L. Zadeh, A simple view of the Dempster-Shafer Theory of Evidence and its implication for the rule of combination, The Al Magazine, Vol. 7, No. 2, pp. 85-90, Summer 1986.

[10] Kari Sentz and Scott Ferson (2002); Combination of Evidence in Dempster-Shafer Theory, Sandia National Laboratories SAND 2002-0835.

[11] Bragge, J.; Korhonen, P., Wallenius, H. and Wallenius, J. (2010). "Bibliometric Analysis of Multiple Criteria Decision Making/Multiattribute Utility Theory". IXX International MCDM Conference Proceedings, (Eds.) M. Ehrgott, B. Naujoks, T. Stewart, and J. Wallenius,. Springer, Berlin 634: 259-268.

[12] P. Sen and J. B. Yang, "Design decision making based upon multiple attribute evaluation and minimal preference information", Mathematical and Computer Modeling, Vol.20, No.3, pp.107-124, 1994.

[13] B. Yang and P. Sen, "Multiple attribute design evaluation of large engineering products using the evidential reasoning approach", Journal of Engineering Design, Vol.8, No.3, pp.211-230, 1997.

[14] Mahmud, T , Hossain, M.S(2012)"An Evidential Reasoning-based Decision Support System to Support House Hunting", International Journal of Computer Applications 57(21):51-58, November 2012.Published by Foundation of Computer Science, New York, USA.

[15] Yang, J. B., Liu, J., Wang, J., Sii, H. S. \& Wang,H.W.(2006) belief rule-base inference methodology using theevidential reasoning approach -RIMER. IEEE Transactions onSystems Man and Cybernetics Part A-Systems and Humans,36, 266-285

[16] D.L. Xu, J. Liu, J.B. Yang, G.P. Liu, J. Wang, I.Jenkinson, J. Ren, Inference and learning methodologyof belief-rule-based expert system for pipeline leak detection,Expert Systems with Applications 32 (2007) 103-113.

[17] B. Yang, J. Liu, D.L. Xu, J. Wang, H.W. Wang, Optimal learning method for training belief rule based systems, IEEE Transactions on Systems, Man, and Cybernetics (Part A) 37 (2007) 569-585.

[18] Patwary, M.J.A, Akter, S, Mahmud, T (2014) “An Expert System to Detect Uterine Cancer under Uncertainty” IOSR Journal of Computer Engineering (IOSR-JCE), e-ISSN: 2278-0661,p-ISSN: 2278-8727, Volume 16, Issue 5, Ver. I (Sep - Oct. 2014), PP 3647)

[19] A. M. Nonvich and T. B. Turksen, "A model for the measuremnt of membership and the consequnces of its empical implementation," Fuzz, Sers Syst., vol. 12, pp. 1-25, 1984.

[20] Mahmud,T.;Rahman,K.N.;Hossain,M.S.(2013).”Evaluation of Job Offers Using The Evidential Reasoning Approach”.Global Journal of Computer Science and Technology. Volume13 Issue 2 Version 1.0 . Published by Global Journals Inc.(USA).

[21] Mahmud,T , Sikder,J (2013) "Intelligent Decision System for Evaluation of Job Offers" $1^{\text {st }}$ National Conferenceon Intelligent Computing and Information Technology(NCICIT ), November 21, CUET, Chittagong-4349,Bangladesh.

[22] Ankita Thakur,1,2 Vijay Mishra,2 and Sunil K. Jain ,Feed Forward Artificial Neural Network: Tool for Early Detection of Ovarian Cancer, Jul 5, 2011.

[23] Yu-Wang Chen, Jian-Bo Yang, Dong-Ling Xu, Zhi-Jie Zhou, Da-Wei Tang, Inference analysis and adaptive training for belief rule based systems, Expert Systems with Applications 38; 12845-12860, 2011

[24] Text book of gynaecology by Dr. D.C.Dutta

[25] Jeffcoats principle of Gynaecology

[26] De Dombal, F. T., Leaper, D. J., Staniland, J.R., Mccann, A. P. \& Horrocks, J. C. Computer-aided diagnosis of acute abdominal pain. British Medical Journal,2(5804) 5 -9, 1972

[27] PREOPERATIVE OVERIAN CANCER DIAGNOSIS USING NEURO-FUZZY APPROACH BY E.O. Madu, V. Stalbovskaya, B. Hamadicharef, E.C. Ifeachor,( Univ. of Plymouth, UK ) S. Van Huffel, and D. Timmerman(KU Leuven,Belgium)

[28] Nicki R. Colledge, Brian R. Walker, Stuart H. Ralstonl, Davidson_s Principles and Practice of Medicine 21st Edition, ISBN-13: 978-0-7020-3084-0, 2010 